\title{
Dynamic 4D Blood Flow Representation in the Aorta and Analysis from Cine-MRI in Patients
}

\author{
M Xavier ${ }^{1}$, A Lalande ${ }^{1}$, PM Walker ${ }^{1}$, C Boichot ${ }^{1}$, A Cochet $^{1}$, O Bouchot $^{2}$, \\ E Steinmetz ${ }^{2}$, L Legrand ${ }^{1}$, F Brunotte $^{1}$ \\ ${ }^{1}$ Laboratoire Electronique, Informatique et Image (LE2I), Université de Bourgogne, Dijon, France \\ ${ }^{2}$ Service de Chirurgie Cardio-Vasculaire, CHU Dijon, France
}

\begin{abstract}
Natural evolution of aortic disease is characterized by a diameter increase that can result in aortic dissection or rupture. Currently the evaluation of risk of rupture or dissection is based on the size of the aorta. However, this parameter is not always relevant and it appears necessary to define new parameters.

In this perspective, $3 D$ velocity imaging acquired with ECG gated velocity-encoded cine-MRI allows the aortic blood flow study. As the acquired images are not directly usable, the present study proposes a $4 D$-representation of aortic blood flow in order to optimize the visualization of the particularities of non-laminar flow within the aorta.

Image were obtained on a $3 T$ imager, and the acquisition plane was orientated perpendicular to the aorta, at the level of the aortic root, thoracic aorta or abdominal aorta according to the pathology. Hence, several blood flow representations (in 2D, 3D or 4D) are provided, and extraction of new parameters (such as maximum velocity, instantaneous blood flow, hemodynamic pressure, backward flow, etc.) are available.
\end{abstract}

\section{Introduction}

In the case of aortic disease, treatment decisions should weigh up the risk of complications caused by the disease against the risk of complications from the operation itself. The maximum diameter of aorta is the principal parameter allowing the evaluation of rupture risk or dissection [1]. However it appears that this parameter is neither reliable nor robust [2] [3].

Therefore, it is necessary to find other evolving criteria to evaluate rupture risk since the only available treatment is surgery. Analysis of blood flow patterns at the level of the aortic disease seems to be promising approach.

Magnetic Resonance Imaging (MRI) is a technique adapted to study aortic blood flow. The present work consists in developing new tools, from cine-MRI, in order to define accurate and useful representations of blood flow in 2,3 or 4 dimensions. From these representations, analysis of a possible parietal stress is feasible. This work allows the extraction of quantitative and representative parameters of blood flow behavior. It is a part of the QIR software ${ }^{1}$ (Quantified Imaging Resource - Université de Bourgogne, Dijon, France) developed in our laboratory.

\section{MR flow imaging}

Images were acquired using ECG gated velocityencoded cine-MRI sequences on a 3T MR imager (Trio TIM, Siemens Medical Solution, Germany) [4]. The acquisition plane was orientated perpendicular to the aorta, at the level of the aortic root, thoracic aorta or abdominal aorta according to the pathology (Figure 1). Acquisition was performed during free-breathing or breath hold according to the localization.

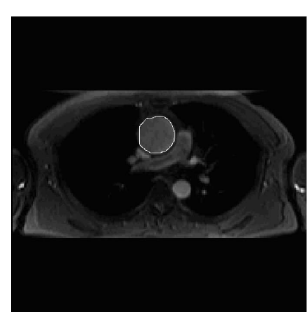

(a)

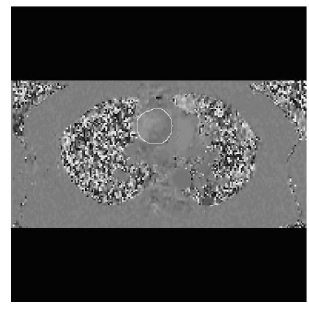

(b)
Figure 1. Flow imaging. (a) Magnitude image and (b) phase contrast image (through plane velocity encoding) at the level of aortic root.

A magnitude image, providing morphological data, and three phase-contrast images (PC images), also called velocity encoded images, were generated during the cardiac cycle with a mean temporal resolution of $35 \mathrm{~ms}$. The three PC images correspond to the velocity encoding in the following three directions: through plane, left-right and anterior-posterior.

\footnotetext{
${ }^{1}$ http://im.le2i.free.fr/.
} 
A particular case of blood flow study illustrates this article. It is the examination of a patient suffering from an ascending aortic aneurysm (maximum diameter : $48 \mathrm{~mm}$ ) with a small regurgitation. The acquisition plane was localized at the level of the aortic root. The acquisition velocities were $100 \mathrm{~cm} / \mathrm{s}$ for Right/Left direction, $100 \mathrm{~cm} / \mathrm{s}$ for Anterior/Posterior direction and $500 \mathrm{~cm} / \mathrm{s}$ for Through Plane. We can see that the velocity values are particularly high in this examination. Magnitude image and PC images were generated during the cardiac cycle (25 images covering the cardiac cycle) with a temporal resolution of $45 \mathrm{~ms}$ and a voxel size of $2 \times 2 \times 5 \mathrm{~mm}$.

\section{Blood flow image processing}

It is unrealistic to achieve an aortic wall detection directly on PC images since the images contain little information on aortic morphology. For this reason, extraction of morphological information from magnitude images had been carried out and combined with functional information (blood flow velocity) from PC images.

\section{Automatic edge detection}

The detection of the aortic wall was performed by an automatic image processing method available irrespective of the localization on the aorta and is detailed in a previous publication [5].

\section{Detection of the aortic lumen}

The automatic detection of the aortic wall defined a region of interest restricted to the aortic lumen on the entire series of magnitude images (Figure 2). On each PC image, restricted to this region of interest, knowledge of the instantaneous velocity for each direction allows the construction of a 3D representation of the aortic blood flow.

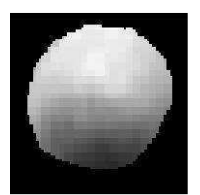

(a)

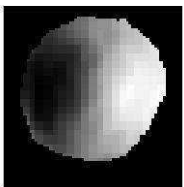

(b)

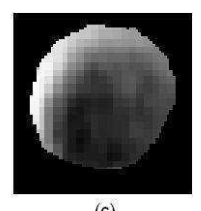

(c)
Figure 2. Phase contrast images of aortic lumen in the three directions ( (a) right-left, (b) anterior-posterior, (c) through plane).

\section{Noise suppression}

A mean filter $(3 * 3)$ was applied to reduce noise in PC images. Indeed noise is partly due to the MR signal artefacts linked to respiratory movement so that signal has to be smoothed. Noise causes difficulties to distinguish one tissue from another (on magnitude image) and to evaluate flow velocities with accuracy. The mean filter is exclusively applied on pixels located in the aortic lumen of PC images to only assess aortic blood signal. Indeed, the automatic detection of aortic wall on magnitude images is robust against noise.

\section{Blood flow velocity decoding}

Blood flow velocity located in a pixel $\left(\mathrm{P}_{i, j}\right)$ depends on acquisition velocity $\left(\mathrm{V}_{\text {Acquisition }}\right)$ and image dynamic ${ }^{2}$ (Dynamic) (4096 gray levels in DICOM [6] velocityencoded images) according to this formula :

Velocity $_{P_{i, j}}=\frac{P_{i, j} \times V_{\text {Acquisition }} \times 2}{\text { Dynamic }}-V_{\text {Acquisition }}(\mathrm{cm} / \mathrm{s})$

Velocity $_{P_{i, j}} \in\left[-V_{\text {Acquisition }},+V_{\text {Acquisition }}\right]$

In our example, $\mathrm{V}_{\text {Acquisition }}$ is equal to $500 \mathrm{~cm} / \mathrm{s}$ for PC images in through plane direction and $\mathrm{P}_{i, j} \in$ $[0$, Dynamic $=4096]$. If $\mathrm{P}_{i, j}=1024$ then Velocity $_{P_{i, j}}=\frac{1024 \times 500 \times 2}{4096}-500=-250 \mathrm{~cm} / \mathrm{s}$

\section{Aortic blood flow analysis}

PC images give us the opportunity to assess 3D blood flow characteristics. A blood flow analysis is also necessary to extract quantitative and qualitative information.

\section{Velocity parameters}

The first parameter extracted is the maximal component of instantaneous velocities in each three spatial directions, giving information about velocity dynamics. An important dynamic, also large velocity variation, in the Right/Left and Anterior/Posterior directions seems to indicate a turbulent flow. And extensive dynamic in the through-plane direction indicates presence of a pulsatile flow.

The maximal 3D blood flow velocity is the maximal norm of the $3 \mathrm{D}$ velocity vectors from all images covering the entire cardiac cycle. A high velocity of 3D flood blow could indicate a problem on aortic valve (in particular in the case of bicuspidy) at the level of aortic root or a loss of elasticity at the level of aortic wall.

\section{Instantaneous blood flow}

Evaluating aortic blood flow during the cardiac cycle may assist the diagnosis of some aortic pathologies. For example, aortic valves disease (especially in case of regurgitation) generates important backflow and consequently a less efficient blood circulation. The instantaneous blood flow is computed from mean instantaneous blood flow velocity and the aortic lumen area.

The mean instantaneous velocity ( Velocity $\left._{\text {Mean }}(t)\right)$ on each image is calculated according to this equation :

$$
\text { Velocity }_{\text {Mean }}(t)=\sum_{i=0}^{\text {Pixel Nb }} \frac{\text { Velocity }_{i}(t)}{\text { Pixel } N b}(\mathrm{~cm} / \mathrm{s})
$$

The lumen area (Area) is computed from the pixel number in the aortic lumen and the pixel size in the image (PixelSurface) in $\mathrm{mm}^{2}$ and :

$$
\text { Area }=\text { PixelSurface } \times 0.01 \times \text { Pixel } N b \quad\left(\mathrm{~cm}^{2}\right)
$$

212 bits allocated for storage in velocity mapping sequence. See field $(0028,0101)$ in DICOM image. 
The instantaneous blood flow $(F l o w(t))$ is the flow at a time $t$ of the cardiac cycle and is defined as :

$$
\text { Flow }(t)=\frac{\text { Area } \times \text { Velocity }_{\text {Mean }}(t) \times 60}{1000} \quad(\mathrm{~L} / \mathrm{min})
$$

\section{Hemodynamic pressure and radial velocity}

The hemodynamic pressure $\left(P_{\text {Bloodi }}(t)\right)$ is the pressure resulting from the heart contraction providing a pulsatile arterial flow. Blood motion creates some pressure on the aortic wall which depends on velocity and blood density $\left(\rho_{\text {Blood }}=1050 \mathrm{~kg} \cdot \mathrm{m}^{-3}\right)$.

$$
P_{\text {Bloodi }}(t)=\frac{1}{2} \times \rho_{\text {Blood }} \times V_{\text {Radiali }}(t)^{2}
$$

To assess hemodynamic pressure applied on the aortic wall, the radial component $\left(\overrightarrow{V_{\text {Radial }}}\right)$ of the instantaneous velocity vector $(\overrightarrow{V(x, y)})$ (close to the aortic wall) must be determined. Important radial velocities might be linked to parietal stress.

In order to calculate a radial component, the center of gravity of the aortic lumen $(G)$ is computed from the outline of aortic lumen. Then, $\left(\overrightarrow{V_{\text {Radial }}}\right)$ is defined as the orthogonal projection of $\left(\overrightarrow{V_{(x, y)}}\right)$ on the line linking the origin of velocity vector $(B)$ and $(G)$ (Figure 3 ).

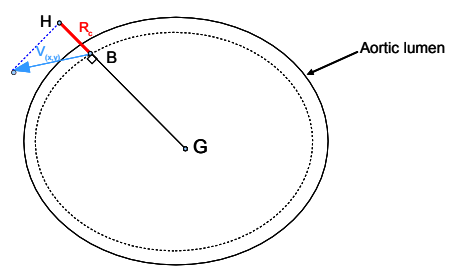

(a)

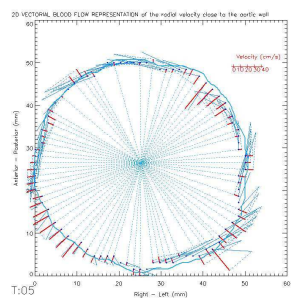

(b)
Figure 3. (a) Radial component of velocity vector - (b) Example of radial velocity display

Backward flow The backward flow (Figure 4) is defined as the volume of blood moving back toward the heart compared with the volume of blood moving away from it. It is computed from the spline curve interpolating flow values $(F l o w(t))$. It is the ratio of the integrals of the curve above and below the $\mathrm{X}$-axis. This parameter is often evaluated in order to determine the regurgitation fraction.

\section{Aortic blood flow representations}

\section{Cine-loop 2D vectorial blood flow diagram}

The cine-loop 2D vectorial blood flow diagram with color coding (according to the velocity orientation) provides a comprehensive and adapted display of flow motion within the plane (Figure 5).
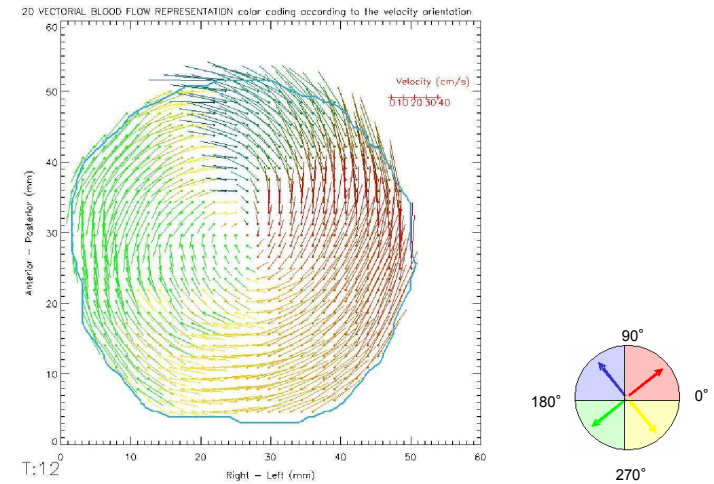

Figure 5. 2D vectorial blood blow representation with color coding according to the velocity orientation at a time $\mathrm{t}=540 \mathrm{~ms}$. Blood flow is driven by a circular motion.

\section{Radial velocity representation}

On each image the hemodynamic pressures were evaluated from radial velocities measured close to the aortic wall (Figure 6).

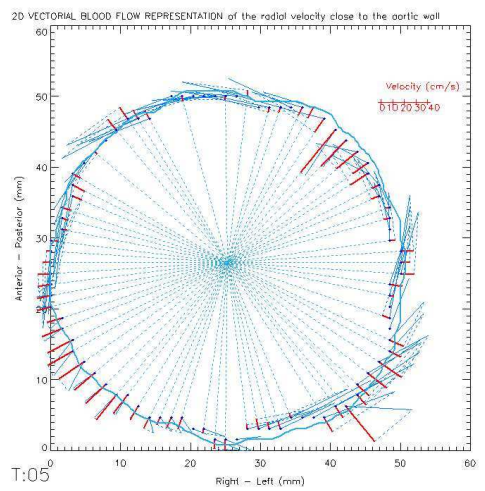

Figure 6. 2D vectorial blood blow representation of radial velocities close to the aortic wall at a time $\mathrm{t}=540 \mathrm{~ms}$.

\section{Planimetric displays}

Planimetric displays were preferred for the vector amplitude of the 3D instantaneous velocity (Figure 7).

\section{Cine-loop 3D vectorial blood flow diagram}

A cine-loop 3D vectorial blood flow diagram with color coding (according to the velocity) provides a comprehensive and adapted display of flow characteristics, especially flow shape and backward flow.

The maximum 3D velocity was deduced from the results obtained on the entire series of images covering a cardiac cycle (Figure 4).

\section{6. $\quad$ Results}

This protocol was applied to patients with aortic disease, such as aortic valve disease, thoracic or abdominal 


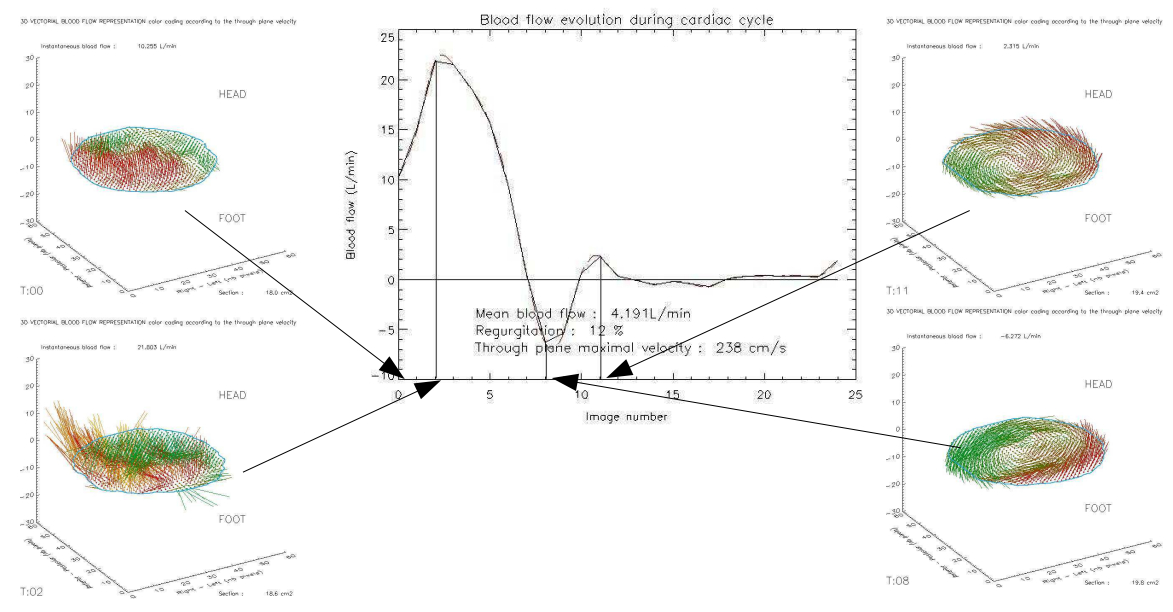

Figure 4. Blood flow evolution during the cardiac cycle

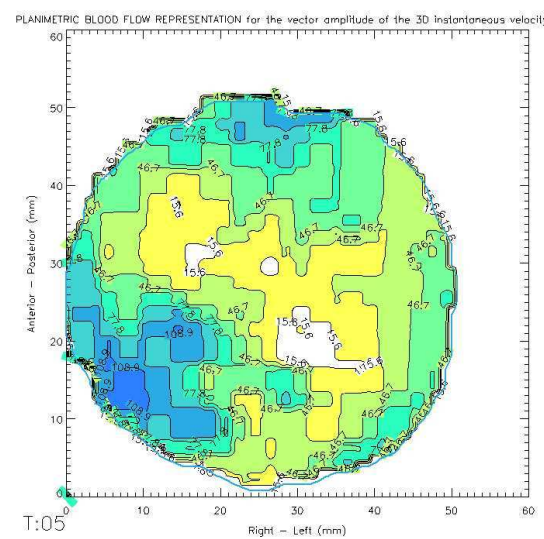

Figure 7. Planimetric representation of 3D instantaneous velocities at a time $\mathrm{t}=540 \mathrm{~ms}$.

aneurysms and tissue disorder affecting aortic elasticity. When making a comparison between the different blood flow representations of patients with various pathologies, graphically, one can observe different behaviors.

Therefore, the population studied is not important enough to provide objective results.

\section{Conclusion}

This work is a preliminary study in order to define a global parameter for detection/classification of aortic pathology. Our 4D representation allows the visualization of various blood flow patterns according to the pathology and the calculation of specific parameters such as absolute maximum velocity and hemodynamic pressures.

It is currently used in two clinical studies (study of small abdominal aortic aneurysms and study of surgical impact in thoracic aorta disease). The impact of surgery on aortic blood flow patterns could be evaluated by comparing the blood flow analysis of pre- and post-operative MRI.

\section{References}

[1] Vorp DA, Raghavan ML, Webster MW. Mechanical wall stress in abdominal aortic aneurysm: influence of diameter and asymmetry. J Vasc Surg 1998;27(4):632-9.

[2] Pitt MP, Bonser RS. The natural history of thoracic aortic aneurysm disease: an overview. J Card Surg 1997;12(2 Suppl):270-8.

[3] Vorp DA, Vande Geest JP. Biomechanical determinants of abdominal aortic aneurysm rupture. Arterioscler Thromb Vasc Biol 2005;25(8):1558-66.

[4] Gatehouse PD, Keegan J, Crowe LA, Masood S, Mohiaddin RH, Kreitner KF, Firmin DN. Applications of phase-contrast flow and velocity imaging in cardiovascular mri. Eur Radiol 2005;15(10):2172-84.

[5] Rose JL, Lalande A, Bourennane E, Walker PM, Bouchot O, Steinmetz E, Legrand L, Voisin Y, Wolf JE, Brunotte F. Automatic Detection of Vessel Wall Contours from CineMri for Aortic Compliance Determination. IEEE Computers in Cardiology 2005;411-414.

[6] Digital imaging and communications in medicine (dicom)part 6: Data dictionary, 2003. URL http://medical. nema.org/dicom/2007/07_06pu.pdf.

Address for correspondence:

Marie XAVIER

Groupe Imagerie Médicale

Le2i, UMR CNRS 5158

Faculté de Médecine / Université de Bourgogne

7 Boulevard Jeanne d'Arc / 21000 Dijon / France

Marie_Xavier@etu.u-bourgogne.fr 